\title{
Bulk Properties of the Matter Produced at Energies of the Beam Energy Scan Program
}

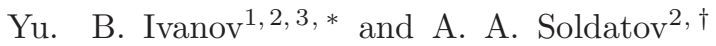 \\ ${ }^{1}$ National Research Centre "Kurchatov Institute", Moscow 123182, Russia \\ ${ }^{2}$ National Research Nuclear University "MEPhI", Moscow 115409, Russia \\ ${ }^{3}$ Bogoliubov Laboratory of Theoretical Physics, JINR, Dubna 141980, Russia
}

\begin{abstract}
Recent STAR data on the bulk observables in the energy range of the Beam-Energy Scan Program at the Relativistic Heavy-Ion Collider are analyzed within the model of the three-fluid dynamics (3FD). The simulations are performed with different equations of state (EoS). The purely hadronic EoS fails to reproduce the data. A good, though imperfect, overall reproduction of the data is found within the deconfinement scenarios. The crossover EoS turns out to be slightly preferable. For this reproduction a fairly strong baryon stopping in the quark-gluon phase is required. The 3FD model does not need two separate freeze-outs (i.e. kinetic and chemical ones) to describe the STAR data. A unified freeze-out is applied at all energies.
\end{abstract}

PACS numbers: 25.75.-q, 25.75.Nq, 24.10.Nz

Keywords: relativistic heavy-ion collisions, hydrodynamics, bulk observables

\section{INTRODUCTION}

Searching for the critical point and phase boundary in the QCD phase diagram is the main motivation for the Beam-Energy Scan (BES) Program at the Relativistic Heavy-Ion Collider (RHIC). Recently, experimental results from the systematic study of bulk properties of the matter produced in $\mathrm{Au}+\mathrm{Au}$ collisions at BES RHIC energies were published by the STAR collaboration [1]. These bulk properties can hardly indicate the presence or absence of the critical point, because the latter are expected to be manifested in enhanced fluctuations in multiplicity distributions of conserved quantities [2, 3]. The systematic study of these bulk properties may reveal the evolution and change in behavior of the system formed in heavy-ion collisions as a function of collision energy. In particular, these bulk observables may indicate if the quark confinement and chiral symmetry breaking at finite baryon densities occur simultaneously or they are separated in the phase diagram as argued in Ref. [4].

In the present paper the bulk observables in the BESRHIC energy range are analyzed within the model of three-fluid dynamics (3FD) [5]. The 3FD model is quite successful in reproduction of the major part of bulk observables at energies of the Alternating Gradient Synchrotron (AGS) at BNL and the Super Proton Synchrotron (SPS) at CERN: the baryon stopping [6, 7], yields of different hadrons, their rapidity [8] and transverse momentum [9] distributions. It is also applicable to the description of the elliptic [10] and directed [11, 12] flow in the AGS-SPS-BES/RHIC energy range. Predictions of the 3FD model for some of the bulk observables at BES-RHIC energies have been already reported in Refs. [7, 9, 12] and fragmentarily compared with pre-

\footnotetext{
*e-mail: Y.Ivanov@gsi.de

†e-mail: saa@ru.net
}

liminary STAR data. The present paper is devoted to the systematic analysis of the final STAR data on the bulk observables.

\section{3FD MODEL}

A specific feature of high-energy heavy-ion collisions is a finite stopping power resulting in a counterstreaming regime of baryon-rich matter of the projectile and target rather then their instantaneous stopping. Within the 3FD description [5] this generally nonequilibrium regime of the baryon-rich matter is modeled by two interpenetrating baryon-rich fluids initially associated with constituent nucleons of the projectile $(\mathrm{p})$ and target $(\mathrm{t}) \mathrm{nu}-$ clei. In addition, newly produced particles, predominantly populating the midrapidity region, are associated with a fireball (f) fluid. Each of these fluids is governed by conventional hydrodynamic equations coupled by friction terms in the right-hand sides of the Euler equations. The hydrodynamic equations do not include viscosity because the dissipation is provided by the friction terms. These friction terms describe energy-momentum loss of the baryon-rich fluids. A part of this loss is transformed into thermal excitation of these fluids, while another part gives rise to particle production into the fireball fluid.

Friction forces between fluids are the key constituents of the model that determine dynamics of the nuclear collision. The friction forces in the hadronic phase were estimated in Ref. [13]. There are no theoretical estimates of the friction in the quark-gluon phase (QGP) so far. Therefore, the friction in the QGP is purely phenomenological. It was fitted to reproduce the baryon stopping at high incident energies within the deconfinement scenarios as described in Ref. [6] in detail.

The physical input of the present 3FD calculations is described in Ref. [6]. The simulations in [6 [12] were performed with different equations of state (EoS's) - a purely hadronic EoS [14] and two versions of the EoS 
involving the deconfinement transition [15], i.e. a firstorder phase transition (1st-order-tr.) and a smooth crossover one. It was demonstrated that the purely hadronic EoS fails to reproduce data at high collision energies $\left(\sqrt{s_{N N}} \gtrsim 5 \mathrm{GeV}\right)$. Therefore, in the present paper we demonstrate results with only these deconfinement EoS's as the most successful in reproduction of various bulk observables at high collision energies and only occasionally present results of the purely hadronic EoS.

Figure 1 illustrates the dynamics of nuclear collisions at BES-RHIC energies. Similarly to Ref. [16], the figure displays dynamical trajectories of the matter in the central box placed around the origin $\mathbf{r}=(0,0,0)$ in the frame of equal velocities of colliding nuclei: $|x| \leq 2 \mathrm{fm}$, $|y| \leq 2 \mathrm{fm}$ and $|z| \leq 2 \gamma_{c m}$ fm, where $\gamma_{c m}$ is the Lorentz factor associated with the initial nuclear motion in the c.m. frame. Initially, the colliding nuclei are placed symmetrically with respect to the origin $\mathbf{r}=(0,0,0) ; z$ is the direction of the beam. The size of the box was chosen to be large enough that the amount of matter in it can be representative to conclude on the onset of deconfinement and to be small enough to consider the matter in it as a homogeneous medium. Nevertheless, the matter in the box still amounts to a minor part of the total matter of colliding nuclei.

Only expansion stages of the evolution are displayed. Evolution proceeds from the top point of the trajectory downwards. Symbols mark the time intervals along the trajectory. Subtraction of the $m_{N} n_{B}$ term from the energy density is done for the sake of suitable representation of the plot. The $\varepsilon-n_{B}$ representation is chosen because these densities are dynamical quantities and, therefore, are suitable to compare calculations with different EoS's. At a given density $n_{B}$, the zero-temperature compressional energy, $\varepsilon\left(n_{B}, T=0\right)$, provides a lower bound on the energy density $\varepsilon$, so the accessible region is correspondingly limited.

The non-equilibrium stage of the expansion is displayed by dashed lines in Fig. 1. The criterion of the thermalization is equality of longitudinal $\left(P_{\text {long }}=T_{z z}\right)$ and transverse $\left(P_{\mathrm{tr}}=\left[T_{x x}+T_{y y}\right] / 2\right)$ pressures ${ }^{1}$ in the box with accuracy better than $10 \%$. Here

$$
T^{\mu \nu} \equiv T_{\mathrm{p}}^{\mu \nu}+T_{\mathrm{t}}^{\mu \nu}+T_{\mathrm{f}}^{\mu \nu}
$$

is the energy-momentum tensor of the composed matter being the sum of conventional hydrodynamical energymomentum tensors of separate fluids. In similar figures of Ref. [6], the onset of equilibration was indicated at earlier stages. The reason is that the condition $\left|P_{\text {long }}-P_{\mathrm{tr}}\right| \leq$ $0.05\left(P_{\text {long }}+P_{\mathrm{tr}}\right)$ indeed is first reached at earlier times as compared with those indicated in Fig. 1. However, after that the difference between $P_{\text {long }}$ and $P_{\text {tr }}$ remains at

\footnotetext{
1 Note that the spatial components of the collective four-velocity of the composed matter in the considered central box are zero due to symmetry reasons.
}
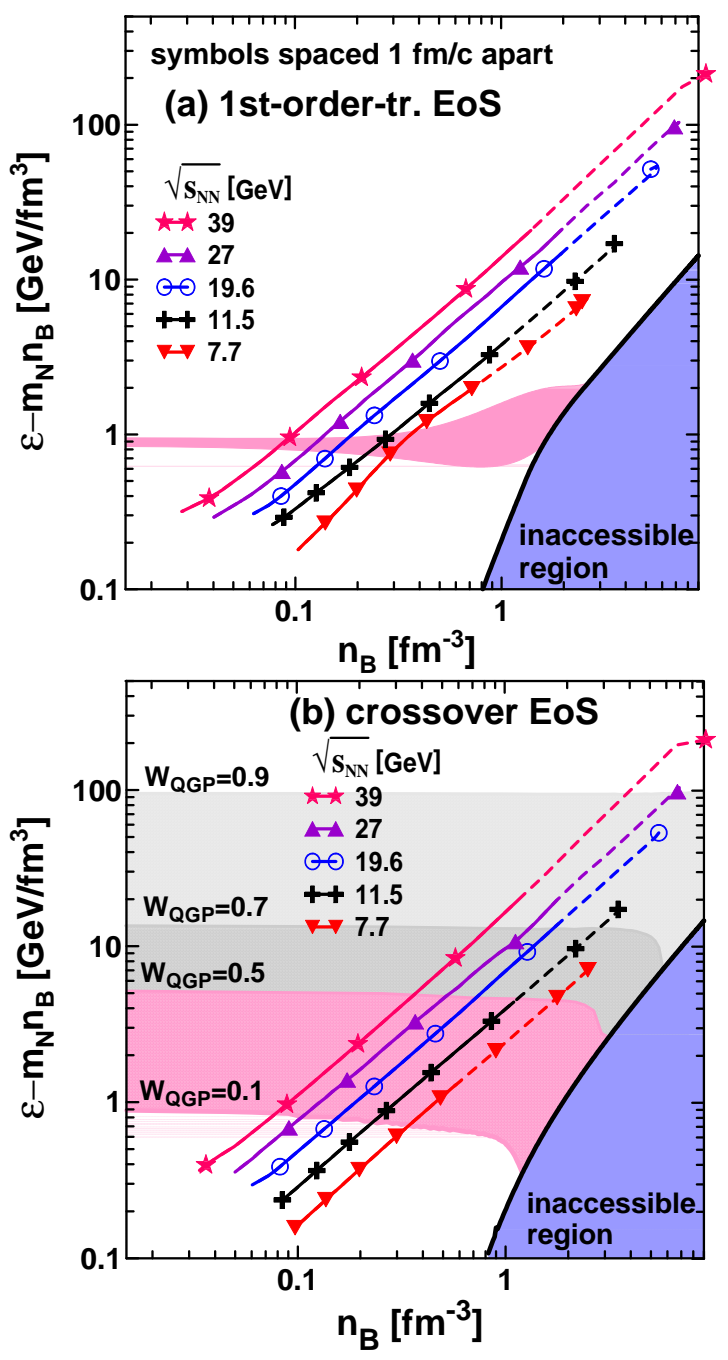

FIG. 1: (Color online) Dynamical trajectories of the matter in the central region of colliding $\mathrm{Au}+\mathrm{Au}$ nuclei $(b=2 \mathrm{fm})$ at BES-RHIC energies. The trajectories are plotted in terms of the proper (i.e. in the local rest frame) baryon density $\left(n_{B}\right)$ and the proper energy density minus $n_{B} m_{N}\left(\varepsilon-m_{N} n_{B}\right)$ where $m_{N}$ is the nucleon mass. Only expansion stages of the evolution are displayed. Symbols on the trajectories indicate the time rate of the evolution: time span between marks is $1 \mathrm{fm} / \mathrm{c}$. Evolution of the equilibrated matter by solid lines, while the stage before the equilibration is displayed by dashed lines. The trajectories are presented for two EoS's: (a) the first-order-transition (1st-order-tr.) EoS and (b) the crossover EoS. For the 1st-order-tr. EoS the mixed phase is displayed by the shadowed region. For the crossover EoS the displayed borders correspond to values of the QGP fraction $W_{Q G P}=$ $0.1,0.5,0.7$ and 0.9 . The inaccessible region is restricted by $\varepsilon\left(n_{B}, T=0\right)-m_{N} n_{B}$ from above.

the $10 \%$ level for some time or even slightly exceeds this $10 \%$ level. Therefore, in the present Fig. 1 we mark the thermalization instant as that after which the difference between $P_{\text {long }}$ and $P_{\mathrm{tr}}$ rapidly drops below the $10 \%$ level.

The trajectories for the first-order-transition and crossover EoS's are very similar, as seen from Fig. 1. 
Note that this similarity is not due to similarity of these two EoS's. This similarity takes place because of the friction forces in the QGP phase that were independently fitted for each EoS [6] in order to reproduce observables in the midrapidity region.

As seen in Fig. 1 the equilibration in the central region is achieved at rather high baryon densities even at high collision energies. This in contrast to conclusions made in Refs. [17, 18] where formation of the initial state for the hydrodynamic evolution was studied. E.g., in Ref. [17] it was even concluded that the stopped nucleons from the target and the projectile end up separated from each other by the distance increasing with the collision energy. For $\sqrt{s_{N N}}>6$ or $10 \mathrm{GeV}$ the latter implies that the created system is not in thermal and chemical equilibrium, and the net-baryon density reached is likely not much higher than that already present in the colliding nuclei [17.

The equilibration in the central region in collisions at energies up to the top SPS one was also analyzed in 19] within the Quark-Gluon String Model (QGSM) and the model of the Ultrarelativistic Quantum Molecular Dynamics (UrQMD). The equilibration time in a small box $(0.5 \mathrm{fm} \times 0.5 \mathrm{fm} \times 0.5 \mathrm{fm})$ within the QGSM and UrQMD [19] is very similar to the 3FD time at the energy of $7.7 \mathrm{GeV}$ (or $30 \mathrm{~A} \mathrm{GeV}$ in the lab. frame). We do not refer to the results of Ref. 19] in the large box ( $5 \mathrm{fm}$ $\times 5 \mathrm{fm} \times 5 \mathrm{fm}$ ) because this box is too large to consider the matter in it as a homogeneous medium in view of the Lorentz contraction of colliding nuclei. However, at the top SPS energy the QGSM and UrQMD equilibration times are essentially longer than that within the $3 \mathrm{FD}$ at the similar energy of $19.6 \mathrm{GeV}$. Consequently, the higher equilibrium densities are reached within the 3FD simulations. This is the effect of the stronger QGP friction required for reproduction of the SPS data [6 9]. Note that even within the hadronic scenario we had to considerably enhance the hadronic friction [5, 6] as compared with its microscopic estimate [13] in order to reproduce data at the top SPS energy.

In spite of the rather high initial baryon densities in the center region even at high collision energies, cf. Fig. 1. the resulting proton rapidity distributions manifest a dip at the midrapidity [6]. In fact, there are two ways to achieve the midrapidity dip in the net-baryon rapidity distribution. The first one is high transparency of colliding nuclei as implemented in Refs. [17, 18]. An alternative scenario is the purely hydrodynamical $1 \mathrm{D}$ expansion of the initially compressed and thermalized thin disk of the baryon-rich matter. In the process of this onedimensional (1D) expansion the baryon charge is pushed out to peripheral rapidities thus producing a dip at the midrapidity. These two scenarios were tested within the 3FD model when the friction parameters were fitted [6] at the BES-RHIC energies. It was found that the problem of the first "transparent" scenario is that too many pions are produced. The actual friction of the 3FD model does not result in an immediate stopping of colliding nuclei, as seen from Fig. 1 E.g., in the initial thermalized state at the collision energy of $39 \mathrm{GeV}$, approximately $30 \%$ of the baryon charge is located in "true" fragmentation regions, i.e. in the baryon-rich matter passed through the interaction region, while $70 \%$ is located in the central fireball 20]. Probably, the "transparent" scenario is relevant at the top RHIC and LHC energies.

\section{BULK OBSERVABLES}

Simulations of $\mathrm{Au}+\mathrm{Au}$ collisions at energies $\sqrt{s_{N N}}=$ 7.7, 11.5, 19.6, 27 and $39 \mathrm{GeV}$ were performed at fixed impact parameters $(b)$. The correspondence between experimental centrality and the mean impact parameter was taken from Glauber simulations of Ref. 21]. Though Ref. 21] deals with higher collision energies, we implemented the reported correspondence to the BES-RHIC energy range in view of practical energy independence of the results deduced in Ref. 21]. If it is not stated otherwise, proton and antiproton observables include contributions from weak decays, while pion and kaon observables do not. This is in agreement with the STAR measurements [1].

\section{A. Transverse-Momentum Spectra}

Figure 2 demonstrates transverse-momentum spectra at the midrapidity of various particles produced in central $(b=2 \mathrm{fm}) \mathrm{Au}+\mathrm{Au}$ collisions at $\sqrt{s_{N N}}=7.7-39 \mathrm{GeV}$ predicted by the 3FD model with three considered EoS's. Experimental STAR data (0-5\% centrality) are from Ref. 1]. As seen, the hadronic scenario fails to describe the data. It fails first only for antiprotons at lower energies and then for all hadrons at higher collision energies.

The first-order-transition and crossover scenarios are quite successful in the reproduction of the data [1] and give very similar results at all considered energies. However, there are exceptions. The $K^{+}$spectra are underestimated at $7.7 \mathrm{GeV}$ and less spectacular at $11.5 \mathrm{GeV}$. This is related to the failure to reproduce the horn in the $K^{+} / \pi^{+}$ratio, discussed below.

A common feature of the $3 \mathrm{FD}$ results is overestimation of high- $p_{T}$ tails of the $p_{T}$ spectra. This overestimation happens earlier (i.e. at lower $p_{T}$ ) for rare probes $\left(\bar{p}\right.$ and $\left.K^{-}\right)$. This is a consequence of the 3FD description being based on grand-canonical statistics which requires large multiplicities to be valid. A lack of exact conservations (of the baryon number and strangeness) in the grand-canonical ensemble results in overestimation of data for rare probes. Even abundant hadronic probes become rare at high momenta. Therefore, their treatment on the basis of the grand-canonical ensemble results in overestimation of their yields. Moreover, as a hadron by itself becomes more rare, the high- $p_{T}$ tail of the spectrum is more strongly suppressed due to restrictions of the canonical ensemble. 


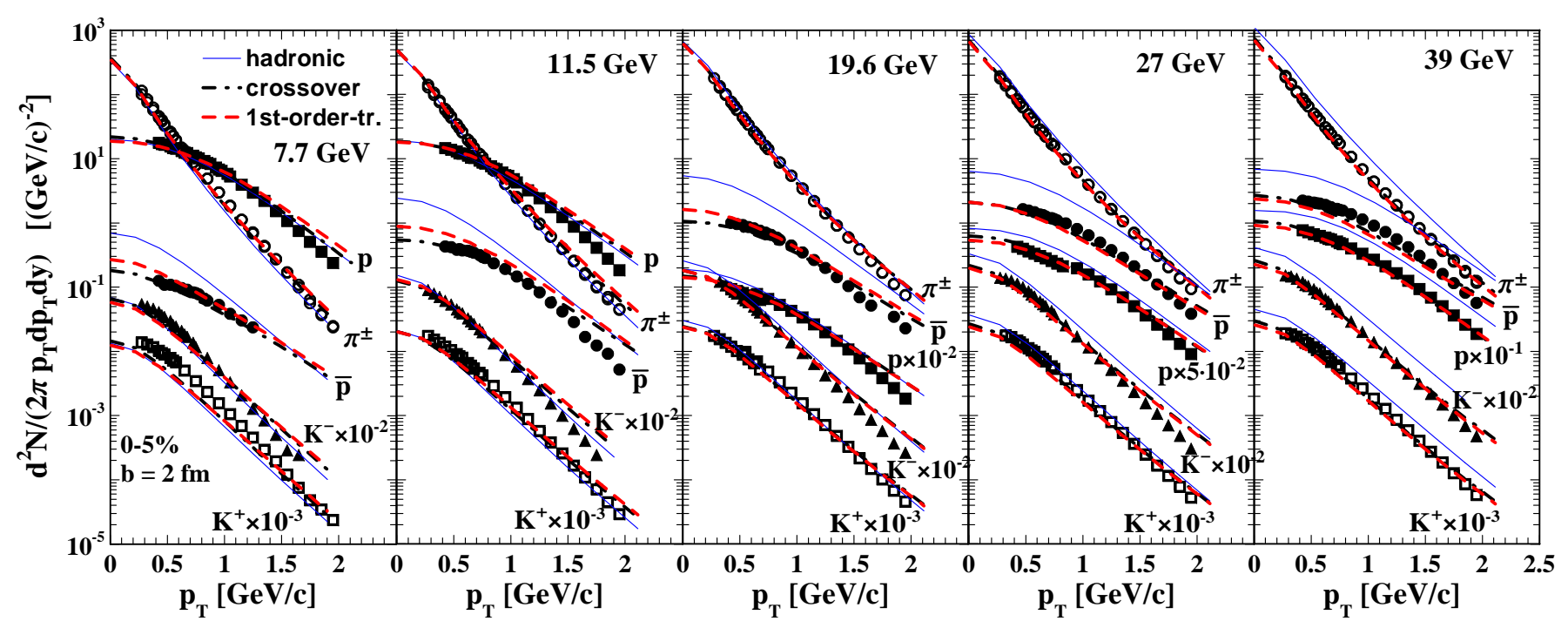

FIG. 2: (Color online) Transverse-momentum spectra at the midrapidity of various particles produced in central $(b=2$ fm) $\mathrm{Au}+\mathrm{Au}$ collisions at $\sqrt{s_{N N}}=7.7-39 \mathrm{GeV}$ predicted by the 3FD model with three considered EoS's. Experimental STAR data (0-5\% centrality) are from Ref. [1].

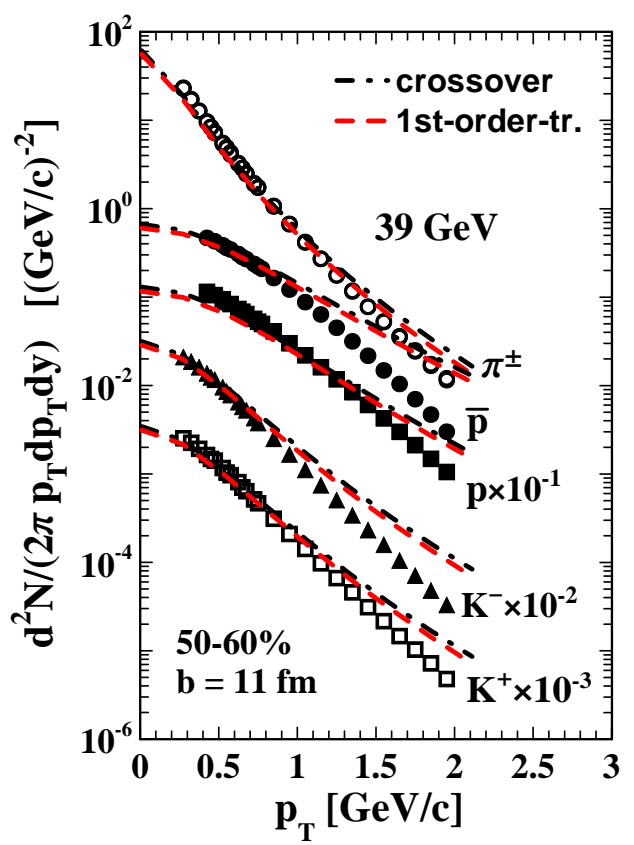

FIG. 3: (Color online) Transverse-momentum spectra at midrapidity of various particles produced in non-central $(b=$ $11 \mathrm{fm}) \mathrm{Au}+\mathrm{Au}$ collisions at $\sqrt{s_{N N}}=39 \mathrm{GeV}$ predicted by the 3FD model with two considered deconfinement EoS's. Experimental STAR data (50-60\% centrality) are from Ref. [1].

Figure 3 confirms our expectations on the applicability of the 3FD model. In peripheral collisions the total number of participants becomes smaller. Therefore, the applicability of the grand-canonical approximation and hence of the 3FD model worsens. It results in earlier (i.e. at lower $p_{T}$ ) overestimation of the tails of the $p_{T}$ spectra. The fact that the 3FD model indeed fails beyond the range of its applicability gives us confidence in the $3 \mathrm{FD}$ results in the range of their applicability.



FIG. 4: (Color online) Mean transverse mass (minus particle mass) at midrapidity for various particles produced in central $(b=2 \mathrm{fm}) \mathrm{Au}+\mathrm{Au}$ collisions as functions of the collision energy. Experimental data are taken from Refs. [22 26] for AGS, 27 30] for SPS and [1] for BES-RHIC energies. 


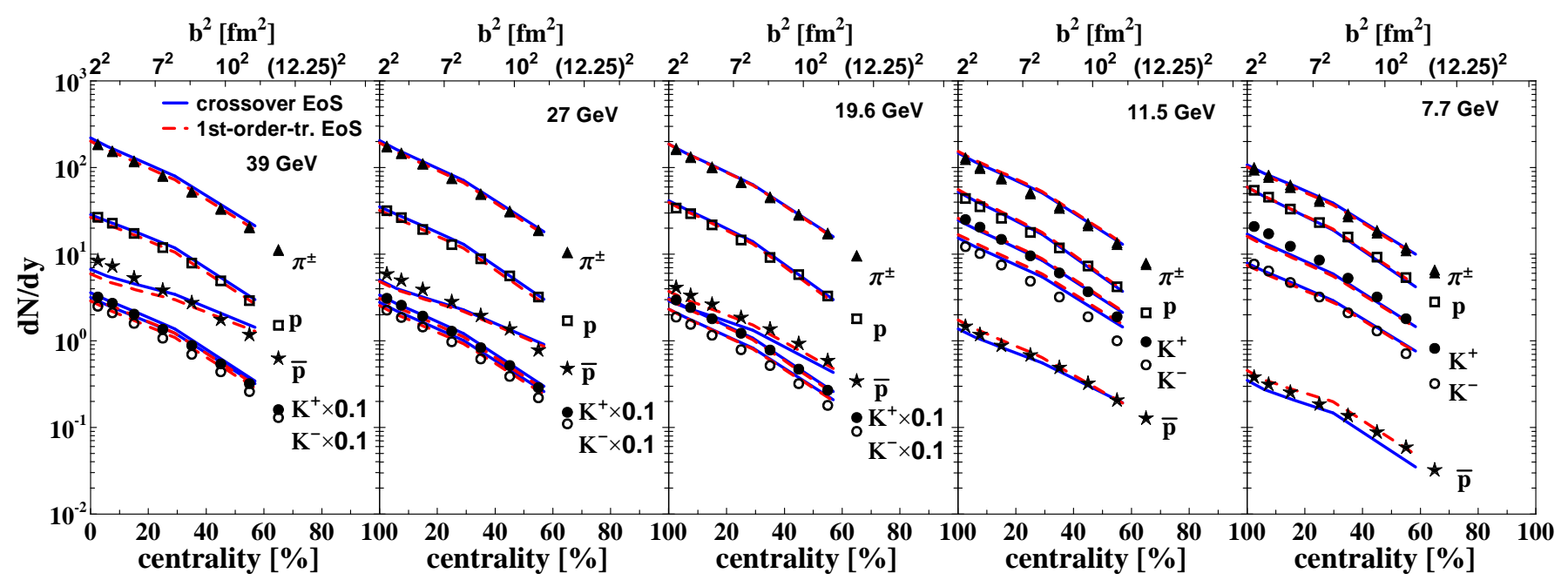

FIG. 5: (Color online) Midrapidity densities $d N / d y$ of various particles produced in $\mathrm{Au}+\mathrm{Au}$ collisions at $\sqrt{s_{N N}}=7.7-39 \mathrm{GeV}$ as functions of the centrality (and impact parameter squared, cf. upper scale) predicted by 3FD model with two considered deconfinement EoS's. Experimental STAR data are from Ref. [1].

\section{B. Mean Transverse Mass}

The collision-energy dependence of the mean transverse mass is an economical way to present the excitation function of the $p_{T}$ spectrum. Excitation functions of the average transverse masses $\left(\left\langle m_{T}\right\rangle-m\right)$ of protons, pions, antiprotons and kaons are presented in Fig. 4. The presented excitation functions are in fact predictions for the BES-STAR data because they were published in Ref. [9] before the STAR data appeared. In Fig. 4 we just compare the predictions of Ref. [9] with the recent STAR data [1]. A detailed discussion of the calculated mean transverse masses is also presented in Ref. [9].

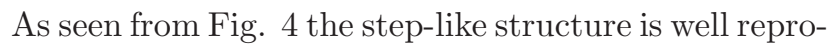
duced within all scenarios, except for antiprotons within the hadronic scenario. This is a consequence the specific freeze-out implemented within the 3FD model [31]. The physical pattern behind this freeze-out resembles the process of expansion of a compressed and heated classical fluid into vacuum, mechanisms of which were studied both experimentally and theoretically. The freeze-out is associated with evaporation from the surface of the expanding fluid and sometimes with explosive transformation of the strongly superheated fluid to the gas. This freeze-out procedure results in a step-like excitation function of the effective freeze-out temperature [9] which in its turn produces this step-like structure in $\left\langle m_{T}\right\rangle$. [32]

Indeed, $\left\langle m_{T}\right\rangle$ can be very approximately presented as

$$
\left\langle m_{T}\right\rangle-m \approx T_{\mathrm{eff}}+\frac{T_{\mathrm{eff}}^{2}}{m+T_{\mathrm{eff}}}
$$

where the inverse slope parameter $T_{\text {eff }}$ can be very approximately related to the freeze-out temperature $T_{\text {frz }}$ and transverse velocity $v_{\text {tr }}$

$$
T_{\text {eff }} \approx T_{\text {frz }}+\frac{1}{2} m v_{\mathrm{tr}}^{2} .
$$

The latter relation results from the nonrelativistic limit of the blast-wave model [33 35]. As seen, $\left\langle m_{T}\right\rangle$ indeed should follow the step-like behavior of $T_{\text {frz }}$ if $v_{\text {tr }}$ is approximately constant. This is actually the case for all particles and scenarios except for the antiprotons within the hadronic scenario. Antibaryons are dominantly produced from the baryon-free f-fluid. Because of long formation time of the f-fluid within the hadronic scenario [6] (as compared with those within the deconfinement scenarios) the transverse velocity $v_{\text {tr }}$ in the f-fluid is not constant but steadily rises with the collision energy, therefore $\left\langle m_{T}\right\rangle$ rises too.

Figure 4 demonstrates that on average the crossover scenario still looks preferable, though the new STAR data are somewhat below those from SPS (for protons and antiprotons). It could seem that the crossover and hadronic scenarios reproduce (or fail to reproduce) the antiproton STAR data to the same extent. However, the slope of the crossover curve is definitely more adequate to the data. In fact, the overestimation of antiproton $\left\langle m_{T}\right\rangle$ is expected because of the overestimation high- $p_{T}$ tails of the $\bar{p}$ spectra discussed above, cf. Fig. 2. The first-order-transition scenario certainly fails for protons in the energy region where onset of deconfinement takes place in this EoS.

\section{Rapidity Densities}

Midrapidity densities of various particles produced in $\mathrm{Au}+\mathrm{Au}$ collisions at $\sqrt{s_{N N}}=7.7-39 \mathrm{GeV}$ as functions of the centrality predicted by $3 \mathrm{FD}$ model are presented in Fig. 5. where they are compared with the recent ex- 


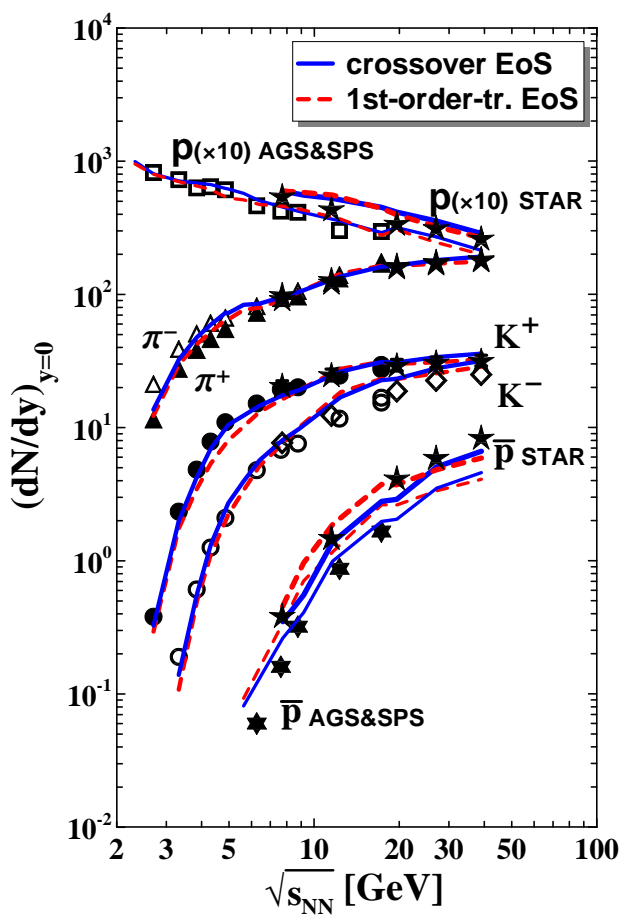

FIG. 6: (Color online) Midrapidity densities $d N / d y$ of various particles produced in central $(b=2 \mathrm{fm}) \mathrm{Au}+\mathrm{Au}$ collisions predicted by $3 \mathrm{FD}$ calculations with two considered deconfinement EoS's as functions of the center-of-mass energy. Thin lines for $p$ and $\bar{p}$ represent densities without contributions of weak decays (corresponding to NA49 data [37, 38]), while thick lines (for $p$ and $\bar{p}$ ) display densities including the weakdecay contributions which are relevant to the STAR data [1]. Experimental data are from compilation of Ref. [36] complemented by recent data from STAR collaboration [1] (stars and open diamonds) and latest update of the compilation of NA49 results [37, 38].

perimental STAR data [1]. The results of calculations are in good overall agreement with the data except for kaons and antiprotons. Figure 6 presents the midrapidity densities of these various particles produced in central $(b=2 \mathrm{fm}) \mathrm{Au}+\mathrm{Au}$ collisions but in a wider energy range $\sqrt{s_{N N}}=2.7-39 \mathrm{GeV}$.

The densities of positive kaons are underestimated at 7.7 GeV and mostly in central collisions, as already seen in $p_{T}$ spectra. This overestimation is not well resolved in Figs. [5 and 6 However, it is clearly visible in the $K^{+} / \pi^{+}$ ratio in Fig. 7 The $K^{-}$densities are overestimated at $\sqrt{s_{N N}} \gtrsim 8 \mathrm{GeV}$, as seen from Fig. 6 and more distinctly from $K^{-} / \pi^{-}$ratio in Fig. 7 As seen from Fig. 2, the soft parts of the $K^{-} p_{T^{-}}$-spectra are reasonably well reproduced at all BES RHIC energies. The overestimation observed in Fig. 6 may result from the overestimation of high- $p_{T}$ tails of $K^{-}$spectra, cf. Fig. 2, which is the common problem for all rare probes described within the approximation of the grand-canonical ensemble. However, this explanation is not applicable at $39 \mathrm{GeV}$ when negative and positive kaons are equally abundant and



FIG. 7: (Color online) The energy dependence of ratios of the midrapidity densities $d N / d y$ : (a) $K^{+}$to $\pi^{+}$and (b) $K^{-}$ to $\pi^{-}$. Compilation of experimental data is from Ref. [38] complemented by recent STAR data [1] and [21] for $\sqrt{s_{N N}}=$ $62 \mathrm{GeV}$ (stars). Calculated ratios with pions including contribution from weak decays are also displayed by thin lines.

the high- $p_{T}$ tail of the $K^{-}$spectrum is quite well reproduced. Another possible source of this overestimation is extrapolation of the experimental $p_{T}$-spectra to a very low $p_{T}$-region in order to calculate the midrapidity density. If this experimental extrapolation differs from the $3 \mathrm{FD}$ prediction for the low- $p_{T}$ spectrum, the difference between the experimental and 3FD midrapidity densities can be substantional.

Antiproton density is a delicate issue. Of course, the above discussed problems of the overestimation of high$p_{T}$ tails and the extrapolation to the low- $p_{T}$ region take place for the antiprotons too. At lower collision energies the 3FD results overestimate the data (cf. Fig. 6), as expected within the approximation of the grand-canonical ensemble. However, at higher energies the data are already underestimated in the central collisions (Figs. 5 and 6) while they reasonably well agree with the 3FD results at larger centralities (impact parameters), see Fig. 5. This is already contrary to our expectations. Recently the production of anti-baryons was studied within the Parton-Hadron-String Dynamics (PHSD) transport approach [39]. It was found that the PHSD results are also 
far from being perfect in reproduction of the antiproton data. This implies that we still do not well understand the mechanism of the $\bar{p}$ production.

The particle ratios, Fig. 7, allow us to more distinctly see the problems in the description of the particle yields. The upper panel of Fig. 7 demonstrates the $K^{+} / \pi^{+}$ratio with the well-known horn structure. Several statistical models [36, 40, 41] have succeeded in reproducing this experimental trend, but they can provide only a statistical description of the heavy-ion collision process. There were no conclusive interpretations of the horn from dynamical approaches. The 3FD model is not an exception, it does not reproduce the horn anomaly in the $K^{+} / \pi^{+}$ratio [8] . Note that only deconfinement transitions are included in the EoS's tested within the 3FD model. Recently, it was suggested that the chiral phase transition within the PHSD model [42] results in a semiquantitative description of the horn anomaly. Restoration of the chiral symmetry enhances the strangeness production. It is equally important that this strangeness enhancement survives in the hadronic phase, as demonstrated in Refs. 42]. Another possible mechanism to explain the "horn" effect was developed in Ref. [43] where the horn anomaly was associated with the occurrence of an anomalous mode for mesons composed of quarks with unequal masses in the course of the Mott transition within the PNJL model. It is still unclear if the produced effect survives in the hadronic phase, i.e. till the freeze-out.

The lower panel of Fig. 7 demonstrates the $K^{-} / \pi^{-}$ratio which is overestimated within the $3 \mathrm{FD}$ model because of too high predicted $K^{-}$yield. In order to demonstrate the effect of the weak decays Figs. 6 and 7 also display results with and without contributions of weak decays at BES-RHIC energies.

Figure 8 demonstrates comparison of the $3 \mathrm{FD}$ results on the $K^{-} / K^{+}$and $\bar{p} / p$ ratios with the corresponding STAR data [1]. The calculated $K^{-} / K^{+}$ratio overestimates the data because of the above mentioned excess of $K^{-}$at $\sqrt{s_{N N}}>8 \mathrm{GeV}$. This is similar to that presented in panel (b) of Fig. 7. The $K^{-} / K^{+}$overestimation at $\sqrt{s_{N N}}=7.7 \mathrm{GeV}$ results from the underestimation of the midrapidity $K^{+}$yield that is also clearly manifested in panel (a) of Fig. 7 The reproduction of the $\bar{p} / p$ ratio [panel (b) of Fig. 8 is quite satisfactory, which is partially the effect of the logarithmic scale of the figure.

\section{SUMMARY}

Recent STAR data [1] on the bulk observables in the BES-RHIC energy range were analyzed within the $3 \mathrm{FD}$ model. The simulations were performed with different equations of state: the purely hadronic EoS [14] and two versions of the EoS involving the deconfinement transition [15], i.e. the first-order phase transition and the smooth crossover one. It was demonstrated that the purely hadronic EoS fails to reproduce the data. Therefore, we focused on the deconfinement EoS's.

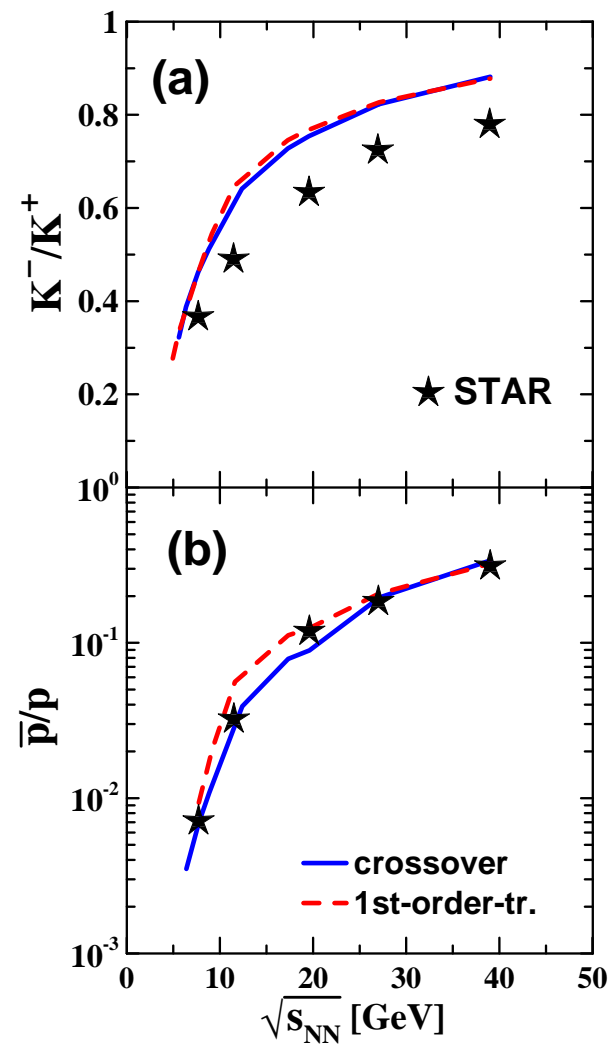

FIG. 8: (Color online) The energy dependence of ratios of the midrapidity densities $d N / d y$ : (a) $K^{-}$to $K^{+}$and (b) $\bar{p}$ to $p$. Experimental STAR data are from Ref. [1].

A good overall reproduction of the bulk observables of Ref. [1] is found within the deconfinement scenarios, of course, within the range of applicability of the 3FD model. Note that some of the discussed 3FD results are in fact predictions because they were published [8, 9] before the appearance of the recent STAR data [1]. The crossover EoS tunes out to be slightly preferable. For this reproduction a quite strong friction in the QGP is required. This friction results in rather high initially equilibrated baryon densities in the central region even at high collision energies. However, this overall reproduction is not perfect. The main problem is the failure to reproduce the horn anomaly in the $K^{+} / \pi^{+}$ratio. It is very probable that this anomaly is a signature of the chiral phase transition [42, 43] which is absent in the present scenarios. It would be instructive to study the effect of the hadronic afterburner at the BES-RHIC energies within the THESEUS event generator [44]. The afterburner may, at least partially, solve the problems with $K^{-}$and $\bar{p}$ observables. It was found earlier [44] that the hadronic afterburner only weakly affects the bulk observables of protons, pions and kaons, though at lower collision energies.

It should be noted that the 3FD model does not need two separate freeze-outs (i.e. kinetic and chemical ones) to describe the BES-RHIC data, as well as the older AGS and SPS data. A unified freeze-out is applied at all ener- 
gies [5, 31]. At the freeze-out stage the baryon-rich fluids are already unified while the net-baryon-free fluid still keeps its identity. Thus, the frozen-out system turns out to be kinetically equilibrated to a good extent, because the relative velocities of the baryon-rich and baryon-free fluids are small [20]. At the same time the chemical equilibrium is absent: two overlapped equilibrated fluids do not provide a chemical equilibrium in the composed system. This way the simultaneous description of $p_{T}$ spectra and hadron abundances is achieved.

\section{Acknowledgments}

This work was carried out using computing resources of the federal collective usage center Complex for simulation and data processing for mega-science facilities at NRC "Kurchatov Institute", http://ckp.nrcki.ru/. Y.B.I. was supported by the Russian Science Foundation, Grant No. 17-12-01427. A.A.S. was partially supported by the Ministry of Education and Science of the Russian Federation within the Academic Excellence Project of the NRNU MEPhI under contract No. 02.A03.21.0005.
[1] L. Adamczyk et al. [STAR Collaboration], Phys. Rev. C 96, no. 4, 044904 (2017) arXiv:1701.07065 [nucl-ex]].

[2] M. A. Stephanov, Phys. Rev. Lett. 102, 032301 (2009) arXiv:0809.3450 [hep-ph]].

[3] F. Karsch and K. Redlich, Phys. Lett. B 695, 136 (2011) arXiv:1007.2581 [hep-ph]].

[4] H. Suganuma, T. M. Doi, K. Redlich and C. Sasaki, J. Phys. G 44, 124001 (2017) arXiv:1709.05981 [hep-lat]].

[5] Yu. B. Ivanov, V. N. Russkikh, and V.D. Toneev, Phys. Rev. C 73, 044904 (2006) nucl-th/0503088.

[6] Yu. B. Ivanov, Phys. Rev. C 87, 064904 (2013) arXiv:1302.5766 [nucl-th]].

[7] Yu. B. Ivanov, Phys. Lett. B 721, 123 (2013) arXiv:1211.2579 [hep-ph]]; Yu. B. Ivanov and D. Blaschke, Phys. Rev. C 92, no. 2, 024916 (2015) arXiv:1504.03992 [nucl-th]].

[8] Yu. B. Ivanov, Phys. Rev. C 87, no. 6, 064905 (2013) arXiv:1304.1638 [nucl-th]].

[9] Yu. B. Ivanov, Phys. Rev. C 89, no. 2, 024903 (2014) arXiv:1311.0109 [nucl-th]].

[10] Yu. B. Ivanov and A. A. Soldatov, Phys. Rev. C 91, no. 2, 024914 (2015) arXiv:1401.2265 [nucl-th]]; Yu. B. Ivanov, Phys. Lett. B 723, 475 (2013) arXiv:1304.2307 [nucl-th]].

[11] V. P. Konchakovski, W. Cassing, Yu. B. Ivanov and V. D. Toneev, Phys. Rev. C 90, no. 1, 014903 (2014) arXiv:1404.2765 [nucl-th]]; Yu. B. Ivanov and A. A. Soldatov, Phys. Rev. C 91, no. 2, 024915 (2015) arXiv:1412.1669 [nucl-th]].

[12] Yu. B. Ivanov and A. A. Soldatov, Eur. Phys. J. A 52, no. 1, 10 (2016) arXiv:1601.03902 [nucl-th]].

[13] L. M. Satarov, Sov. J. Nucl. Phys. 52, 264 (1990).

[14] V. M. Galitsky and I. N. Mishustin, Sov. J. Nucl. Phys. 29, 181 (1979).

[15] A. S. Khvorostukhin, V. V. Skokov, K. Redlich, and V. D. Toneev, Eur. Phys. J. C48, 531 (2006) nucl-th/0605069.

[16] I. C. Arsene, L.V. Bravina, W. Cassing, Yu.B. Ivanov, A. Larionov, J. Randrup, V.N. Russkikh, V.D. Toneev, G. Zeeb, D. Zschiesche, Phys. Rev. C 75, 034902 (2007) nucl-th/0609042.

[17] A. Bialas, A. Bzdak and V. Koch, arXiv:1608.07041 [hep$\mathrm{ph}]$.

[18] C. Shen and B. Schenke, arXiv:1710.00881 [nucl-th].

[19] L. V. Bravina et al., Phys. Rev. C 78, 014907 (2008) arXiv:0804.1484 [hep-ph]].

[20] Y. B. Ivanov and A. A. Soldatov, Phys. Rev. C 97,
021901(R) (2018) arXiv:1711.03069 [nucl-th]].

[21] B. I. Abelev et al. [STAR Collaboration], Phys. Rev. C 79, 034909 (2009) arXiv:0808.2041 [nucl-ex]].

[22] L. Ahle et al. [E866 and E917 Collaborations], Phys. Lett. B 476, 1 (2000) nucl-ex/9910008.

[23] J. L. Klay et al. [E895 Collaboration], Phys. Rev. Lett. 88, 102301 (2002) nucl-ex/0111006.

[24] J. Barrette et al. [E877 Collaboration], Phys. Rev. C 62, 024901 (2000) nucl-ex/9910004].

[25] L. Ahle et al. [E802 Collaboration], Phys. Rev. C 60, 064901 (1999).

[26] L. Ahle et al. [E802 Collaboration], Phys. Rev. C 57, 466 (1998).

[27] S. V. Afanasiev et al. [NA49 Collaboration], Phys. Rev. C 66, 054902 (2002) nucl-ex/0205002.

[28] C. Alt et al. [NA49 Collaboration], Phys. Rev. C 77, 024903 (2008) arXiv:0710.0118 [nucl-ex]].

[29] C. Alt et al. [NA49 Collaboration], Phys. Rev. C 73, 044910 (2006).

[30] T. Anticic et al. [NA49 Collaboration], Phys. Rev. C 69, 024902 (2004).

[31] V. N. Russkikh and Yu. B. Ivanov, Phys. Rev. C 76, 054907 (2007) nucl-th/0611094; Yu. B. Ivanov and V. N. Russkikh, Phys. Atom. Nucl. 72, 1238 (2009) arXiv:0810.2262 [nucl-th]].

[32] B. Mohanty, J. e. Alam, S. Sarkar, T. K. Nayak and B. K. Nandi, Phys. Rev. C 68, 021901 (2003) nucl-th/0304023.

[33] J. P. Bondorf, S. I. A. Garpman and J. Zimanyi, Nucl. Phys. A296, 320 (1978).

[34] P. J. Siemens and J. O. Rasmussen, Phys. Rev. Lett. 42, 880 (1979).

[35] E. Schnedermann, J. Sollfrank and U. W. Heinz, Phys. Rev. C 48, 2462 (1993) nucl-th/9307020.

[36] A. Andronic, P. Braun-Munzinger and J. Stachel, Nucl. Phys. A 772, 167 (2006) nucl-th/0511071.

[37] C. Blume, M. Gazdzicki, B. Lungwitz, M. Mitrovski, P. Seyboth, and H. Strobele, Compilation of NA49 Numerical Results, http://edms.cern.ch/document/1075059

[38] C. Blume and C. Markert, Prog. Part. Nucl. Phys. 66, 834 (2011) arXiv:1105.2798 [nucl-ex]].

[39] E. Seifert and W. Cassing, arXiv:1710.00665 [hep-ph].

[40] A. Andronic, P. Braun-Munzinger and J. Stachel, Phys. Lett. B 673, 142 (2009) Erratum: [Phys. Lett. B 678, 516 (2009)] arXiv:0812.1186 [nucl-th]]. 
[41] K. A. Bugaev, D. R. Oliinychenko, J. Cleymans, A. I. Ivanytskyi, I. N. Mishustin, E. G. Nikonov and V. V. Sagun, EPL 104, no. 2, 22002 (2013) arXiv:1308.3594 [hep-ph]].

[42] A. Palmese, W. Cassing, E. Seifert, T. Steinert, P. Moreau and E. L. Bratkovskaya, Phys. Rev. C 94, no. 4, 044912 (2016) arXiv:1607.04073 [nucl-th]]; W. Cassing, A. Palmese, P. Moreau and E. L. Bratkovskaya,
Phys. Rev. C 93, 014902 (2016) arXiv:1510.04120 [nuclth]].

[43] A. Dubinin, A. Radzhabov, D. Blaschke and A. Wergieluk, Phys. Rev. D 96, 094008 (2017) arXiv:1608.05383 [hep-ph]].

[44] P. Batyuk et al., Phys. Rev. C 94, 044917 (2016) arXiv:1608.00965 [nucl-th]]. 